\title{
High Humidity and Age-Dependent Fruit Susceptibility Promote Development of Trichothecium Black Spot on Apple
}

Pengbo Dai, Xiaofei Liang, Yajing Wang, State Key Laboratory of Crop Stress Biology in Arid Areas and College of Plant Protection, Northwest A\&F University, Yangling, Shaanxi, 712100, China; Mark L. Gleason, Department of Plant Pathology and Microbiology, Iowa State University, Ames, IA 50011, U.S.A; and Rong Zhang and Guangyu Sun, ${ }^{\dagger}$ State Key Laboratory of Crop Stress Biology in Arid Areas and College of Plant Protection, Northwest A\&F University, Yangling, Shaanxi, 712100, China

\begin{abstract}
Fruit bagging is a widely used orchard practice in China. Trichothecium black spot (TBS) is a disease highly associated with the fruit bagging. In this study, we characterized in vitro factors affecting the causal agent, Trichothecium roseum, and TBS development and infection histology on field-bagged apple fruit in situ. Under in vitro conditions, conidial germination required exogenous nutrients, and the germination rate was significantly promoted by high humidity, a condition mimicking the bag microenvironment. Germ tubes penetrated fruit via natural openings including stomata, lenticels, and surface cracks. To determine the chronology of infection by $T$. roseum, 'Fuji' fruit were inoculated in the field at

different developmental stages. The earliest infection occurred 60 days after full bloom (dafb), and disease incidence increased as fruit maturity advanced. At harvest time (165 dafb), lesions on more recently inoculated fruit (105 dafb, $150 \mathrm{dafb})$ were larger than lesions from fruit inoculated on earlier dates. Histological observation showed that infection of younger fruit elicited stronger host lignification responses restricting lesion development. Taken together, our results support the hypothesis that high humidity in sealed bags and increased susceptibility associated with advancing fruit maturity are key factors promoting $T$. roseum infection and TBS symptom development on bagged apple fruit.
\end{abstract}

Fruit bagging, which is a common practice in China to produce blemish-free apple fruit, entails sealing young fruit ( 2 to $3 \mathrm{~cm}$ in diameter) in paper or plastic bags until near maturity (Fig. 1). By providing physical protection, fruit bagging not only improves fruit appearance by promoting skin coloration but also protects fruit against damage from pesticide residues, insects, diseases, bird pecks, and mechanical abrasion from hailstones (Feng et al. 2014; Wen and Ma 2006). This practice was first introduced from Japan in the 1990s, and has become widespread in the last 20 years in China. It is now applied to over $70 \%$ of apple orchards in China. With the proliferation of fruit bagging, pests such as Carposina niponensis Walsingham, Adoxophyes orana Fischer von Roslerstamm, and infectious apple diseases such as ring rot, bitter rot, and apple scab, were controlled efficiently in China (Wang et al. 2006).

However, fruit bagging has resulted in new disease problems, including black spot (Wang et al. 2006). Black spot is caused by the fungi Trichothecium roseum (Pers.) Link (Guo et al. 2005), Alternaria tenuissima (Nees) Wiltshire, Acremonium sclerotigenum (Moreau \& R. Moreau ex Valenta) W. Gams (Wang et al. 2014), and Acremonium stritum (Pers.) Link (Guo et al. 2005). Among these pathogens $T$. roseum is one of the most important, causing 1- to 6mm-diameter circular black spots in the fruit surface, with most spots located on the epicarps surrounding sepals (Fig. 2) (Chen et al. 2002); this form of black spot is called Trichothecium black spot (TBS). A similar disease called pink rot caused by $T$. roseum was reported on bagged apple fruit in Japan (Yukita and Kuradate 1988).

Black spot occurs widely in Shaanxi, Hebei, and Shandong provinces ( $\mathrm{Li}$ et al. 2015). The incidence ranged from 10 to $30 \%$ among orchards in the region of Yangtai and Shandong, and reached up to 90\% in some orchards in 2011 (Wang et al. 2014). Black spot had

${ }^{\dagger}$ Corresponding author: G. Y. Sun; E-mail: sgy@nwsuaf.edu.cn

Funding: This research was supported by National Natural Science Foundation of China, project no. 31772113; China Agriculture Research System, project no. CARS-27; and Shaanxi Provincial Science Research and Development Program (2016KTCQ02-14).

Accepted for publication 30 July 2018.

(c) 2019 The American Phytopathological Society a higher severity in cv. Fuji fruit, and relative lower severity in early-maturing varieties, such as cvs. Pink Lady, Gala, and Jonagold in the field (Guo 2013; Liu et al. 2007). Black spot caused significant economic losses by degrading fruit appearance and reducing storage life (Li et al. 2015; Liu et al. 2007).

Factors affecting black spot occurrence included climate conditions, fruit bag types, and humidity inside fruit bags. Li et al. (2015) reported that the amount of rainfall in August and September correlated positively with black spot incidence. Fruit in plastic bags, with presumably less air circulation and higher humidity than in paper bags, tended to have higher incidence (Chen et al. 2002; Guo et al. 2005; Liu et al. 2007). Furthermore, decreasing humidity in plastic bags by pricking some holes in them reduced black spot incidence (Chen et al. 2002).

Understanding TBS biology is critical for effective disease control. The objective of this study was to characterize the infection biology of $T$. roseum and to identify key host, pathogen, and environmental factors affecting TBS development.

\section{Materials and Methods}

Inoculum preparation. The Trichothecium roseum (Pers.) Link isolate TR45, obtained from symptomatic apple fruit in Yangling, Shaanxi Province, China, was identified by morphology and molecular phylogenetic analysis, and its pathogenicity was verified according to Koch's postulate. Isolate TR45 was used to produce a GFP strain used in this study. GFP expression was achieved by protoplast transformation with the GFP expression vector pRM7, where GFP was driven by the constitutive RP27 promoter. This GFP strain was maintained on potato dextrose agar (PDA).

To harvest conidia, 6-day-old PDA cultures grown under $25^{\circ} \mathrm{C}$ in darkness were flooded with $10 \mathrm{ml}$ of sterile water, scraped, and filtered through one layer of Miracloth (Calbiochem, USA). The conidial suspension was adjusted to a concentration of $5 \times 10^{6} \mathrm{CFU} \mathrm{m}{ }^{-1}$ and supplemented with Tween-80 to a final concentration of $0.1 \%$ for inoculation purposes.

Effects of exogenous nutrients, humidity, and darkness on in vitro conidial germination. To test the effect of exogenous nutrients on conidial germination, a $20-\mu 1$ drop of conidial suspension $\left(10^{6}\right.$ $\mathrm{CFU} \mathrm{ml} \mathrm{m}^{-1}$ ) was placed on a glass slide using a micropipette, airdried for 10 to 15 min under air flow, and amended with a 30- $\mu$ l drop of sterile water or 1,10 , or $100 \%$ apple juice. For each treatment, 
drops were placed separately on the two ends and the middle of a glass slide. After the glass slide containing solution drops was placed in a petri dish containing approximately $20 \mathrm{ml}$ of water agar, the petri dish was sealed by paraffin film. To test the effect of humidity on germination, three $20-\mu l$ drops of conidial suspension $\left(10^{6} \mathrm{CFU} \mathrm{m}{ }^{-1}\right)$ were pipetted separately onto the two ends and middle of glass slides, then $30-\mu l$ drops of $10 \%$ apple juice were added to each droplet, and the mixtures were air-dried for 10 to $15 \mathrm{~min}$ under air flow, and sealed in petri dishes or chambers with relative humidity $(\mathrm{RH})$ of $100,99,97,95,92$, or $85 \%$. Relative humidity levels of 100,99 , 97 , or $95 \%$ were achieved by amending approximately $20 \mathrm{ml}$ of aqueous agar with $0,0.3,0.9$, or $1.5 \mathrm{~mol} \mathrm{NaCl}$, respectively, in sealed 85-mm-diameter dishes (Harris et al. 1970; Wang et al. 2015; Xu et al. 2001). The glass slides were placed in the lid of petri dishes that contained agar supplemented with $\mathrm{NaCl}$. In contrast, $\mathrm{RH}$ levels of 92 and $85 \%$ were achieved by saturated $\mathrm{KNO}_{3}$ and $\mathrm{KCl}$ solutions, respectively, in glass chambers (Kosuge and Hewitt 1964). Slides were incubated on platforms that were $2 \mathrm{~cm}$ above the surface of the salt solutions. To test the effect of illumination on conidial germination, $20-\mu 1$ droplets of conidial suspension $\left(10^{6} \mathrm{CFU} \mathrm{ml}^{-1}\right)$ in $10 \%$ apple juice were deposited on the ends and middle of glass slides by a micropipette, then placed in the lids of petri dishes containing approximately $20 \mathrm{ml}$ of aqueous agar to maintain $100 \% \mathrm{RH}$. Petri dishes were then sealed immediately with paraffin film, and either placed in an illumination incubator with constant light (cold white light; illuminance $=15,000 \mathrm{Lx}$ ) or wrapped in black plastic bags to ensure darkness. All treatments were incubated at $25^{\circ} \mathrm{C}$. Thirty-six glass slides were used per treatment. The tested glass slides were taken from the incubators and observed for conidial germination by fluorescent microscopy at $3,6,9,12,15,18,24,36,48,72,96$, and $120 \mathrm{~h}$ postinoculation (Olympus BX51, Japan; X-Cite 120Q). A conidium was considered germinated when the germ tube was longer than half of the length of the conidium. Three slides were used for counts at each observation time. Germination rates were calculated based on examination of at least 250 conidia. The experiments were each performed three times.

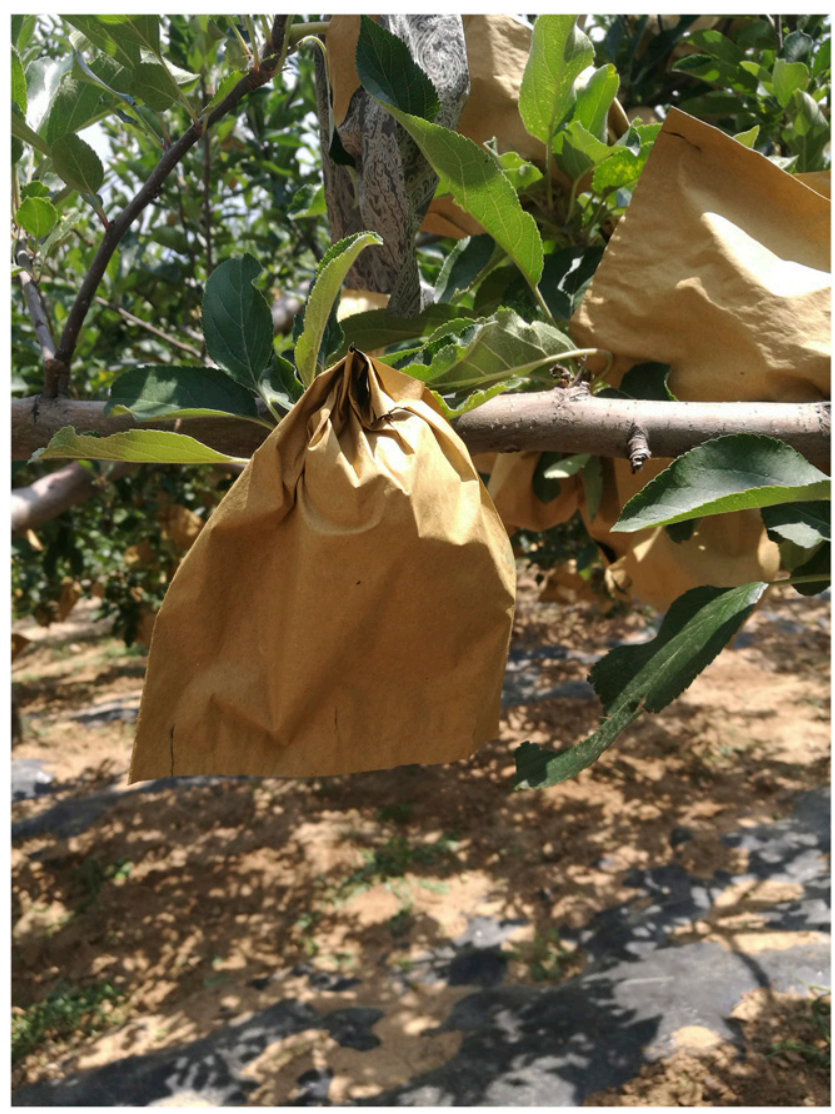

Fig. 1. Young apples are covered with fruit bags at $3-4 \mathrm{~cm}$ in diameter after bloom.
In vitro inoculation. To determine penetration sites of $T$. roseum, apple fruit (cv. Fuji) were harvested at 30, 45, 75, 120, and 150 days after full bloom (dafb) for laboratory inoculations. After harvested apples were sprayed with $75 \%$ ethanol and air-dried, about $1 \mathrm{ml}$ of inoculum suspension $\left(5 \times 10^{6} \mathrm{CFU} \mathrm{ml}{ }^{-1}\right)$ was sprayed uniformly on the entire apple surface. For each developmental stage, 24 fruit of similar size were inoculated, and an additional five fruit were mock inoculated with sterile distilled water. Inoculated apples were placed in plastic boxes (eight fruit per $40 \times 28 \times 12 \mathrm{~cm}$ box). The sterile gauze moistened with $50 \mathrm{ml}$ sterile water was placed in the bottom of each plastic box to maintain high relative humidity $(\mathrm{RH}=98 \pm$ $1 \%$ ). Boxes with inoculated apples were kept at $25 \pm 1{ }^{\circ} \mathrm{C}$ in darkness. Apple peels $\left(1 \times 1 \mathrm{~cm}^{2}\right)$ were sampled for microscopic examination at $0,6,12,18,24,36,48$, and $72 \mathrm{~h}$ postinoculation (hpi). For each sample point, three apples were taken from the box and examined using fluorescent microscopy (Olympus BX51, Japan; X-Cite 120Q). Five peel segments of each apple were examined. Each developmental stage had three replicates. The experiment was conducted in 2015 and repeated in 2016.

Scanning electron microscopy. Apple peels containing germinated conidia identified by fluorescent microscopy were further sampled for scanning electron microscope observation. The peels $(5 \times$ $5 \mathrm{~mm}^{2}$ ) were fixed in $4 \%$ glutaraldehyde solution for $24 \mathrm{~h}$. The samples were washed three times with a phosphate buffer, then dehydrated in a series of aqueous-ethanol solutions $(50,70,85,95$, and $100 \%$ ). Subsequently, samples were dried in a critical point dryer under $\mathrm{CO}_{2}$ and mounted with double-adhesive tape onto aluminum stubs and coated with gold. Samples were examined using a scanning electron microscope (Model S-4800, Hitachi, Japan). For each developmental stage, peels from three apples were examined and five peel segments were sampled per fruit.

Field inoculation and microscopic observation. Field inoculation in 2015 and 2016 was carried out in a block of 7-year-old trees (cv. Fuji) on M.9 rootstock in an apple orchard located in Yangling. Inoculations were performed in situ on fruit at $30,45,60,75,105$, or 150 dafb. Fungicides were not applied in the experimental plots. For each inoculation scheme, 30 asymptomatic apples were randomly selected from five trees and were inoculated in the same way as in vitro inoculation. In addition, 10 control fruit per developmental stage were mock-inoculated with $0.1 \%$ Tween-80. All inoculations were done near sunset. After inoculation, the apples were covered with wet two-layer paper bags (Jingguo, Jinggong Ltd., China). TBS incidence was investigated 15 days after inoculation; incidence of TBS was calculated as follows:

$$
\begin{aligned}
\text { Incidence } \%= & (\text { number of diseased fruit } / \text { total number of fruit }) \\
& \times 100
\end{aligned}
$$

Fruit showing TBS symptoms were harvested from the orchard; black spot tissues were excised. Slices were made perpendicular to the fruit surface by free hand, and examined under light and fluorescent microscopy.

Statistical analysis. Variance of incidence of TBS on apples for each fruit developmental stage and year was calculated using the "general linear model - univariate" function in IBM SPSS Statistics 19 at $P=0.05$ level. Effect of apple age and year on diameter of TBS lesions was also analyzed as described above.

\section{Results}

Effects of exogenous nutrients, relative humidity, and darkness on in vitro conidial germination of $T$. roseum. At $12 \mathrm{hpi}$, germination rate of $T$. roseum in sterile distilled water was $2 \%$, which increased to $\sim 20 \%$ by $72 \mathrm{hpi}$. At $12 \mathrm{hpi}$, germination rates in 1,10 , and $100 \%$ apple juice were $5 \pm 0.8 \%, 68.8 \pm 8.8 \%$, and $98.7 \pm$ $1.5 \%$, respectively (Fig. 3A). At 9 hpi, nearly $100 \%$ of conidia had germinated at $\mathrm{RH}=100,99$, or $97 \%$. At $\mathrm{RH}=95 \%$ and $\mathrm{RH}=$ $92 \%, 100 \%$ germination was observed at $18 \mathrm{hpi}$ and $48 \mathrm{hpi}$, respectively (Fig. 3B). Conidial germination was completely inhibited at $\mathrm{RH}=85 \%$. 
Constant light delayed germination by $\sim 2 \mathrm{~h}$ compared with complete darkness (Fig. 3C).

Penetration of fruit in vitro by $T$. roseum. Conidial germination began at $9 \mathrm{hpi}$, and germination rate steadily increased. Conidia surrounding lenticels were more likely to germinate than those located further from lenticels. At $24 \mathrm{hpi},>80 \%$ of the conidia near lenticels (within a radius of $200 \mu \mathrm{m}$ ) germinated, whereas the germination rate was $10 \%$ for those located at greater distances (Fig. 4 A-C).

Germinated conidia penetrated apple fruit via natural openings. In early stage fruit (75 dafb), penetration mainly occurred through stomata (Fig. 4E). For fruit at 120 dafb, germinated conidia mostly penetrated via lenticels (Fig. 4D). For fruit at 150 dafb, most conidia near lenticels did not germinate or penetrate the lenticels, presumably because the lenticels had become heavily lignified, as evidenced by strong autofluorescence (Fig. $4 \mathrm{G}$ ). $T$. roseum conidia also penetrated the fruit surface via microcracks in the epicuticular wax layer (Fig. 4F).

Infection of fruit in field trials. The incidence of black spots at 15 days postinoculation (dpi) was calculated in 2015 and 2016. Apple age significantly affected incidence of black spots $(P=0.003)$ (Table 1). The effect of year was not significant $(P=0.553)$ (Table 1); hence, the data sets for the 2 years were pooled and analyzed (Fig. 5A). TBS symptoms did not show up on fruit that had been inoculated at $30 \mathrm{dafb}$ or $45 \mathrm{dafb}$. For fruit inoculated at $60 \mathrm{dafb}$, disease incidence per fruit was about $40 \%$, and the number of black spots per symptomatic fruit ranged from two to five. For fruit inoculated at $75 \mathrm{dafb}$, disease incidence was about $60 \%$, and the number of black spots per symptomatic fruit ranged between 2 and 15 (P. Dai, unpublished data). At 150 dafb, disease incidence increased to $90 \%$, and the number of black spots per symptomatic fruit ranged from 2 to 11 (P. Dai, unpublished data).

At harvest maturity (165 dafb), we also quantified TBS lesion diameter on fruit. Apple age significantly affected diameter of black spots $(P=0.010)$ (Table 2$)$. The effect of year was not significant $(P=0.182)$ (Table 2$)$, and hence the 2 years of data were pooled together and analyzed (Fig. 5B and C). In general, fruit inoculated at later maturity dates produced larger TBS lesions. Lesion diameters for fruit inoculated at 60 and 75 dafb ranged from $500 \mu \mathrm{m}$ to $3 \mathrm{~mm}$, but most were smaller than $2 \mathrm{~mm}$. For fruit inoculated at $105 \mathrm{dafb}$, lesion diameter ranged from 1 to $6 \mathrm{~mm}$, and $>90 \%$ were between 1.5 and $4 \mathrm{~mm}$ (Fig. 5B). For fruit inoculated at $150 \mathrm{dafb}$, lesion diameter ranged from 1 to $7 \mathrm{~mm}$, and most were larger than $2 \mathrm{~mm}$ (Fig. 5B and C). The average diameter of fruit inoculated at 105 or $150 \mathrm{dafb}$ was significantly larger than for fruit inoculated at 60 to 75 dafb (Fig. 5B).

Histological changes in fruit response to infection at different fruit developmental stages. Inoculation at 75 dafb induced approximately $800-\mu \mathrm{m}$-diameter TBS lesions at $15 \mathrm{dpi}$. At the lesion sites, epidermal, hypodermal, and parenchymal cells were observed to have changed color, which was interpreted as cell death. Hyphal colonization, however, was limited to the epidermal and hypodermal cell layers (Fig. 6A). At 30 to $40 \mathrm{dpi}$, lesion sizes had expanded to around $1,400 \mu \mathrm{m}$ in diameter, but hyphal colonization was still restricted mainly to the epidermal and hypodermal cell layers, and hyphal extension into dead parenchymal cell layers was observed rarely (Fig. 6B). At 50 to 70 dpi, infectious hyphae began to decline as evidenced by decreased green fluorescence. Hyphae in the inner tissue layer appeared to decline first, leaving remnant green fluorescence

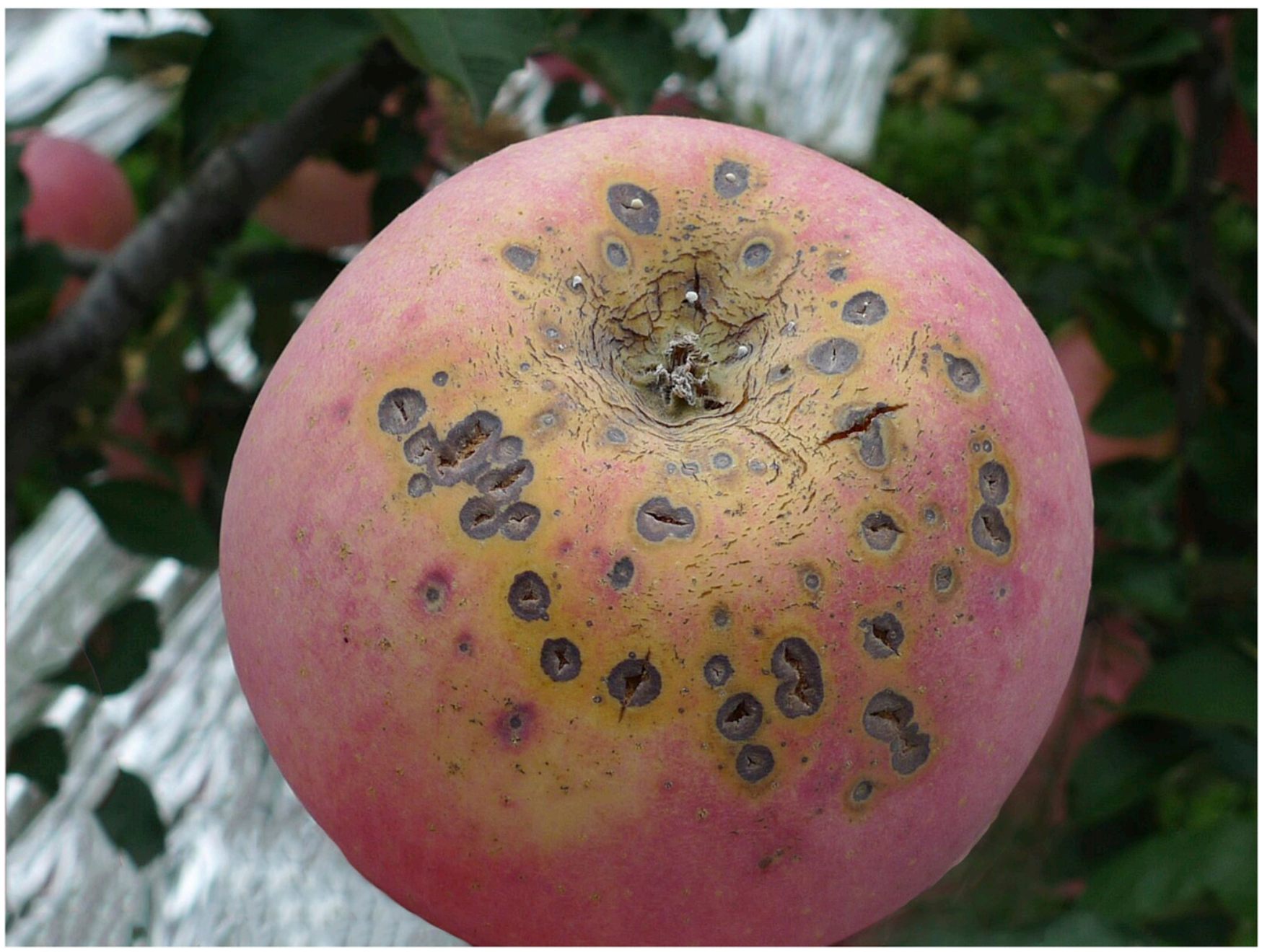

Fig. 2. Apple with Trichothecium black spot after removing bag in field. 
at the penetration sites (Fig. 6C). In certain sections, green fluorescence disappeared completely, and a yellow fluorescent zone surrounding lesions was observed, possibly due to lignification (Fig. 6D).

Early colonization events ( 15 and $30 \mathrm{dpi}$ ) of $T$. roseum on fruit inoculated at 105 dafb were similar to those inoculated at 75 dafb, except that the colonized area was larger (Fig. 6E and F). At 50 to 60 dpi, significant hyphal colonization of parenchymal cells was observed (Fig. 6G), and no hyphal death or lignification zone formation was observed in $>20$ independent section samples.
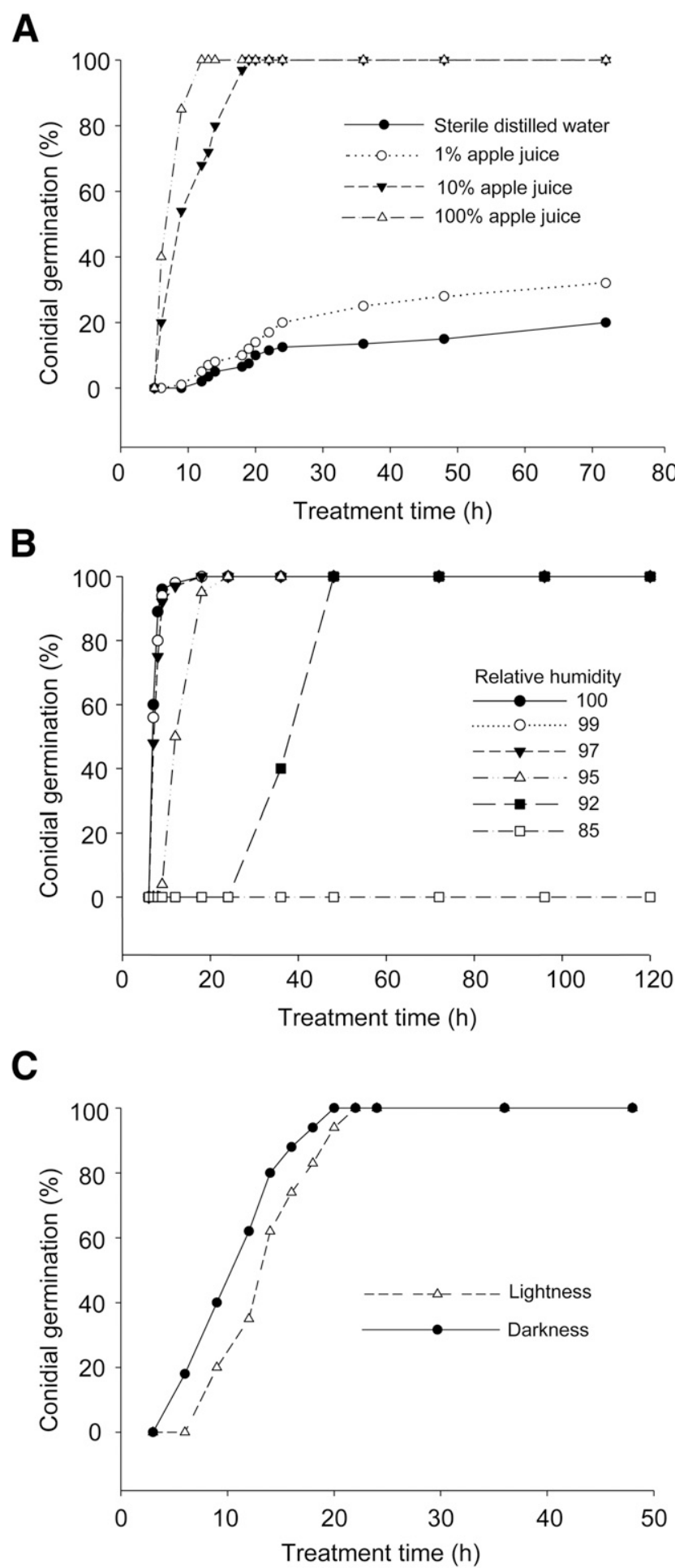

Fig. 3. Effects of nutrition (A), humidity (B), and light conditions (C) on in vitro conidial germination of Trichothecium roseum. Apple juice solution (10\%) was used as the nutrient source to assess the effects of humidity and light. For each treatment, germination rate was calculated based on at least 250 conidia.
For fruit inoculated at $150 \mathrm{dafb}$, intense colonization of parenchymal cells was obvious as early as 15 dpi (Fig. $6 \mathrm{H})$.

At harvest maturity (165 dafb), a layer separating TBS lesions from healthy fruit tissue was obvious with fruit that had been inoculated at $60 \mathrm{dafb}$, but not when inoculation occurred on fruit that were nearly mature (150 dafb) (Fig. 7A and B). Phloroglucinol staining, indicating lignin accumulation, was observed in the separation layer on young fruit (60 dafb), but not on mature fruit (150 dafb) (Fig. 7C and D).

\section{Discussion}

TBS is an emerging disease in China that degrades the appearance and storage time of field-bagged apple fruit. Our results showed that a favorable microenvironment and fruit age-dependent susceptibility were key factors promoting TBS disease development.

On fruit surfaces, conidia near lenticels had much higher germination rates than conidia far away from lenticels. We demonstrated that the availability of exogenous nutrients is of critical importance for $T$. roseum conidial germination based on an in vitro assay. Many sugars and organic acids, including glucose, fructose, sucrose, malic acid, and citric acid, can be exuded around lenticels during fruit expansion (Belding et al. 2000; Liu and Xiao 2005; Wrona and Grabowski 2004). We speculate that $T$. roseum might rely on lenticel-derived nutrients for conidial germination and subsequent fruit infection.

The moist microenvironment inside fruit bags could be a factor promoting conidial germination of $T$. roseum. Li et al. (2008) measured the dynamic diurnal $\mathrm{RH}$ changes inside fruit bags from late June to late August, discovering that RH inside fruit bags stayed above $97 \%$ for more than $10 \mathrm{~h}$ at night. Furthermore, when a small amount of rainfall occurred (e.g., $0.2 \mathrm{~mm}$ during $1 \mathrm{~h}$ ), RH inside fruit bags stayed above $97 \%$ for around $12 \mathrm{~h}$. Li et al. (2008) proposed that $\mathrm{RH}$ inside fruit bags could reach $97 \%$ for $10 \mathrm{~h}$ or longer at night or in association with rainfall periods. In the present study, we characterized the effect of humidity on conidial germination of $T$. roseum. At $25^{\circ} \mathrm{C}$, in free water or at $\mathrm{RH}>97 \%$, most conidia germinated within 9 $\mathrm{h}$, whereas at RH of 95 or $92 \%, 12$ or $40 \mathrm{~h}$, respectively, were needed for $50 \%$ of conidia to germinate. These results suggest that RH levels inside fruit bags often satisfy requirements for T. roseum conidial germination and infection. Several investigations showed that apple fruit enclosed in plastic bags had higher incidence of black spot than fruit in paper bags (Chen et al. 2002; Guo et al. 2005; Liu et al. 2007). We speculate that the reason for this difference is that the high levels of RH associated with $T$. roseum activity occur more commonly in plastic than in paper fruit bags.

Occurrence of TBS is influenced by fruit developmental stage. According to our data on infection sites and hyphal extension at different fruit ages, we divided TBS occurrence into three stages. The first stage, at 30 to $45 \mathrm{dafb}$, resulted in no $T$. roseum infections, and microscopic examination showed that most conidia on the surface of these fruit did not germinate. Zhang et al. (2010) reported that there were no detectable soluble nutrients on apple fruit surfaces before $45 \mathrm{dafb}$. We speculate that a lack of diffusible nutrients limited conidial germination. At the second stage (60 to $75 \mathrm{dafb}$ ), hyphae of $T$. roseum penetrated apple epicarps via stomata on the fruit surface; most black spots were relatively small $(<2 \mathrm{~mm})$ and they did not expand. Microscopic examination showed that hyphae were restricted from spreading into the parenchymal cell layers, and the hyphae eventually died. Lesion borders were clearly confined by a separation zone containing heavily lignified cells. At this stage, low $\mathrm{pH}$ and high levels of antimicrobial substances such as phenolics and acids in apple fruit might retard hyphal extension (Hwang 1983; Kohn and Hendrix 1983; Sitterly and Shay 1960). With further fruit expansion, most stomata transformed into lenticels, and some microcracks appeared on fruit surfaces. The third stage (105 to $150 \mathrm{dafb}$ ) entails lenticel and microcrack infection. At these fruit ages, hyphae penetrated apple epicarps via lenticels or microcracks on fruit surfaces, and most black spots were relatively large (3 to $6 \mathrm{~mm}$ in diameter). Microscopic examination showed that fungal hyphae spread into the parenchymal cell layer more extensively than at earlier fruit ages, and there was no evidence of hyphal death or separation zones. At 
these stages, ripening fruit undergo a series of changes such as $\mathrm{pH}$ and soluble nutrient increase and decline in concentration of antimicrobial substances (Hwang 1983; Kohn and Hendrix 1983; Sitterly and Shay 1960), which might reduce fruit defense reactions and stimulate hyphal extension.

T. roseum can cause apple fruit rot during storage (Hamid et al. 2014; Kwon et al. 2014). We inferred that hyphae of T. roseum in black spots might contribute to T. roseum fruit rot in storage. For
Table 1. Analysis of variance of incidence of Trichothecium black spot on apples at different apple ages and in different years

\begin{tabular}{lccc}
\hline Factors & df $^{\mathbf{a}}$ & $\boldsymbol{F}$-value & $\boldsymbol{P}$ value \\
\hline Age of apples $^{\mathrm{b}}$ & 5 & 74.517 & 0.003 \\
Year $^{\mathrm{c}}$ & 1 & 0.492 & 0.533 \\
\hline
\end{tabular}

${ }^{a}$ Degrees of freedom. $P$ value indicated significance at 0.05 level.

${ }^{b}$ Inoculation was done at 30, 45, 60, 75, 105, 150 days after full bloom.

${ }^{\mathrm{c}}$ Experiment was conducted in 2015 and 2016.

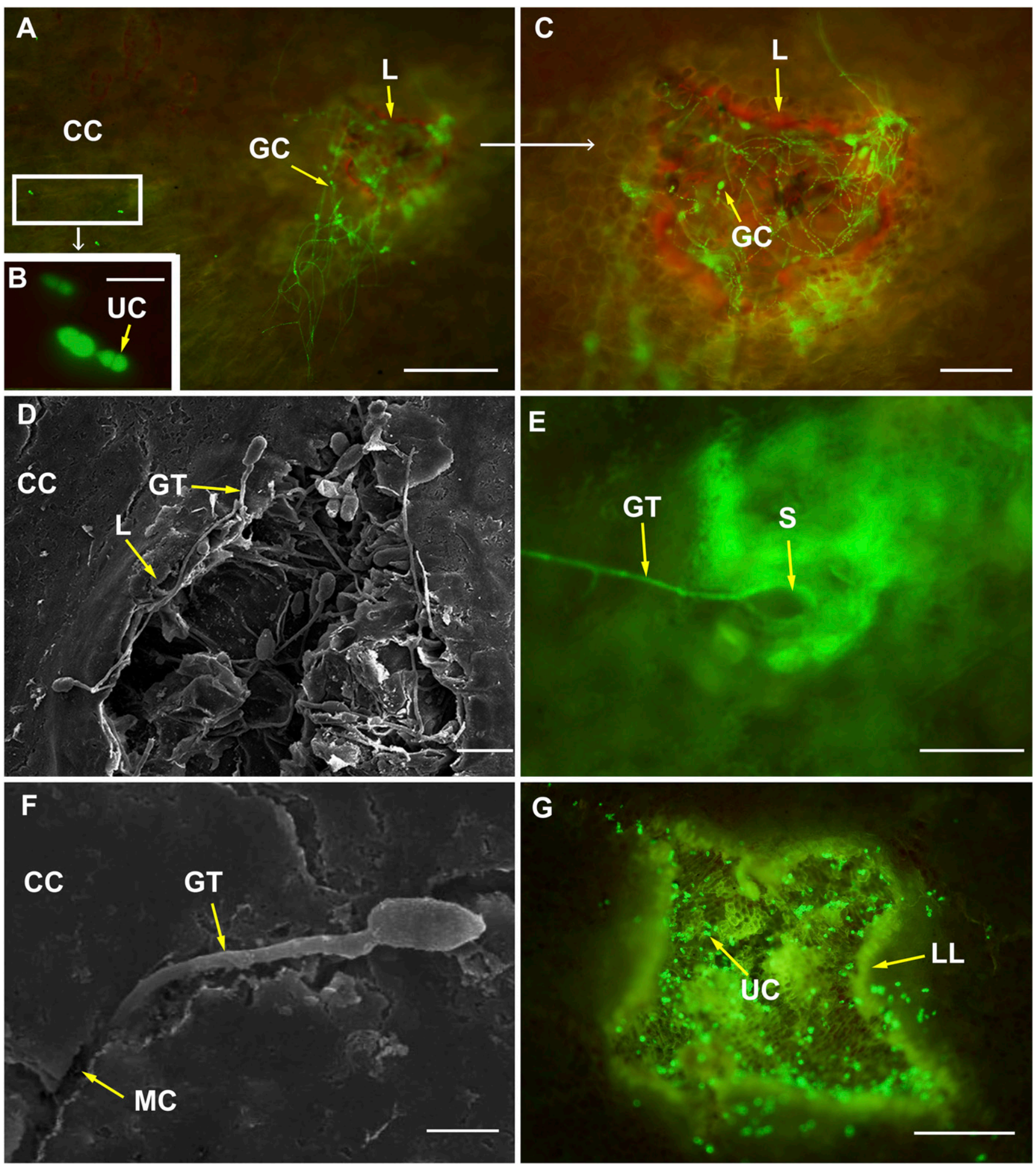

Fig. 4. Conidia germination and entrance of Trichothecium roseum on apple fruit. A, B, C, D, Conidia on fruit surface tend to germinate in the vicinity of the lenticel site, but not on cuticle-coated area (white box in A, B). E, Stomata penetration of $T$. roseum germ tube on apple that was inoculated 75 days after full bloom. F, Penetration of microcrack in the epicuticular wax layer on an apple that was inoculated 150 days after full bloom. G, Lenticels of fruit that were inoculated 150 days after full bloom exhibited strong autofluorescence, and the inoculated conidia failed to germinate within 10 days. GC: germinated conidia; UC: ungerminated conidia; GT: germ tube; CC: cuticle-coated area; L: lenticel; LL: lignified lenticel; S: stoma; MC: microcrack. Bar (A and G) $=200 \mu \mathrm{m}, \operatorname{Bar}(C)=100 \mu \mathrm{m}, \operatorname{Bar}(B$ and F) $=20 \mu \mathrm{m}, \operatorname{Bar}(D$ and $E)=50 \mu \mathrm{m}$. 
the stomatal infection type, $T$. roseum hyphae were dead in black spots or constrained by the host's lignified zone. We speculate that these early-fruit-stage black spots might not extend or cause fruit rot at storage time. For lenticel and microcrack infections, hyphae remained viable in black spots. We speculate that these black spots could cause fruit rot during cold storage. Moreover, we also found that apple fruit with no black spot symptoms at harvest produced TBS during storage. Therefore, we speculate that $T$. roseum formed quiescent infections in microcracks or nonlignified lenticels on apple fruit surface that could lead to fruit rot after harvest.

Bagging protects apple fruit from pests and diseases, but also creates microenvironments that can favor development of TBS. As bagging blocks access by fungicide sprays, it poses challenges for chemical management of TBS. Based on our results that RH inside fruit bags is important for conidial germination, we suggest reducing RH inside bags and in orchards to suppress TBS. A first step would

Table 2. Analysis of variance of diameter of Trichothecium black spot on apples at different apple ages and in different years

\begin{tabular}{lccc}
\hline Factors & df $^{\text {a }}$ & $\boldsymbol{F}$-value & $\boldsymbol{P}$ value \\
\hline Age of apples $^{\mathrm{b}}$ & 3 & 30.460 & 0.010 \\
Yearc $^{\mathrm{c}}$ & 1 & 2.994 & 0.182 \\
\hline
\end{tabular}

a Degrees of freedom. $P$ value indicated significance at 0.05 level.

b Inoculation was done at 30, 45, 60, 75, 105, 150 days after full bloom.

c Experiment was conducted in 2015 and 2016.
A

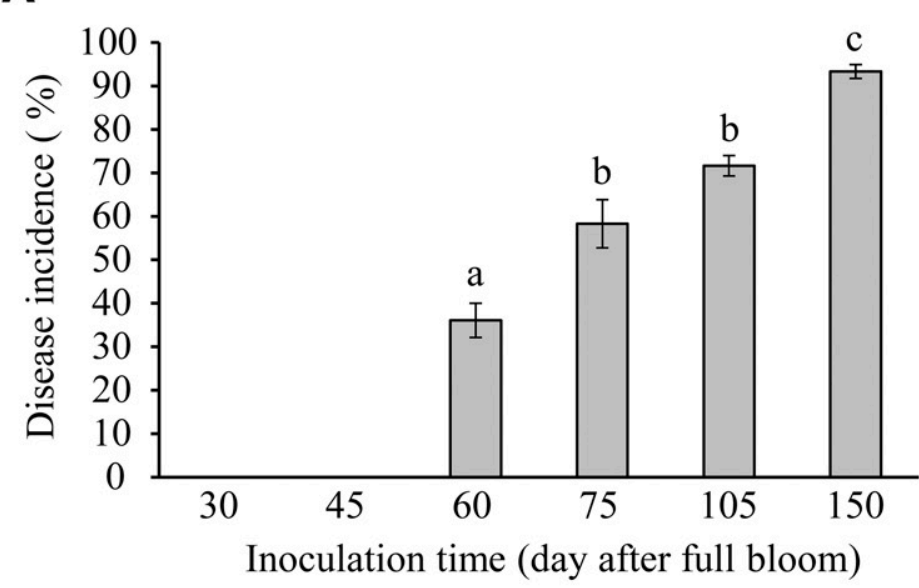

B

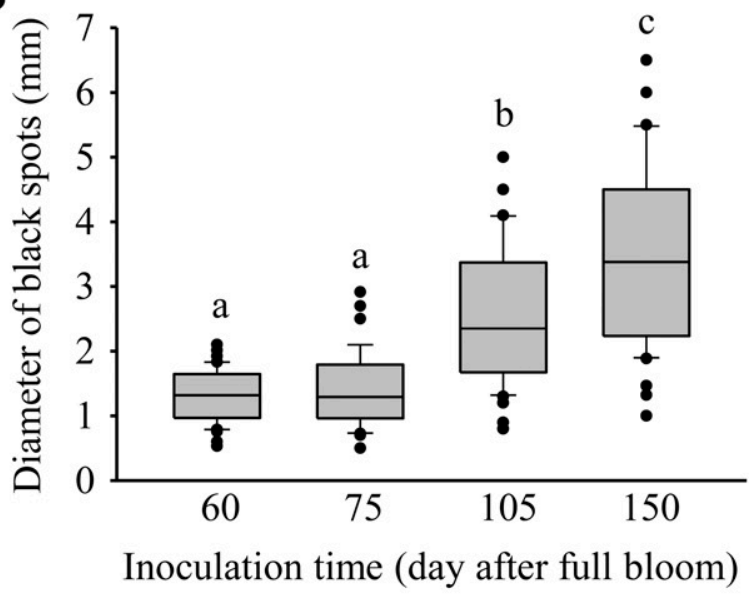

C

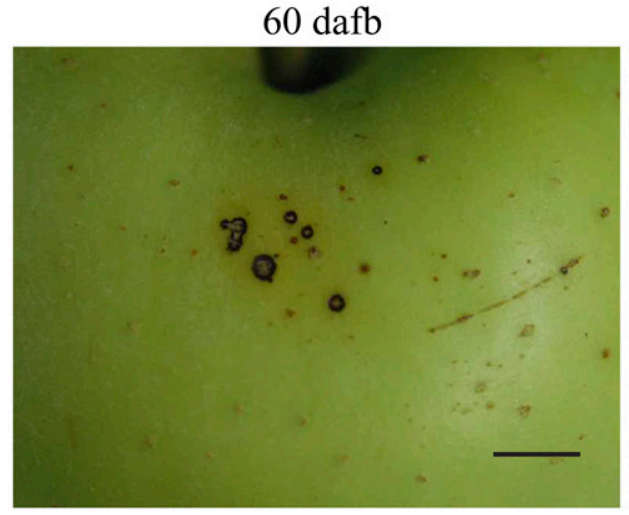

$105 \mathrm{dafb}$

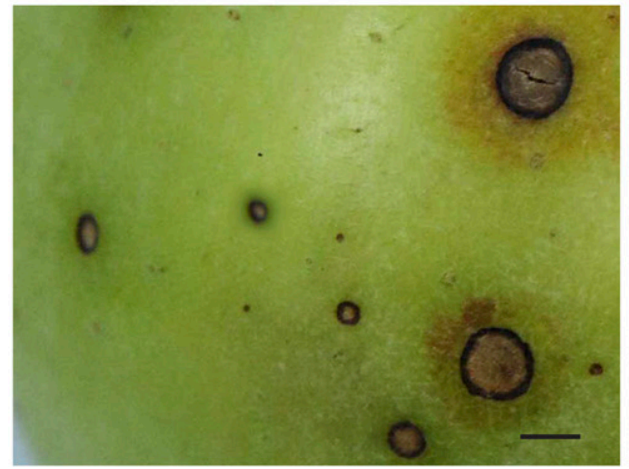

$75 \mathrm{dafb}$

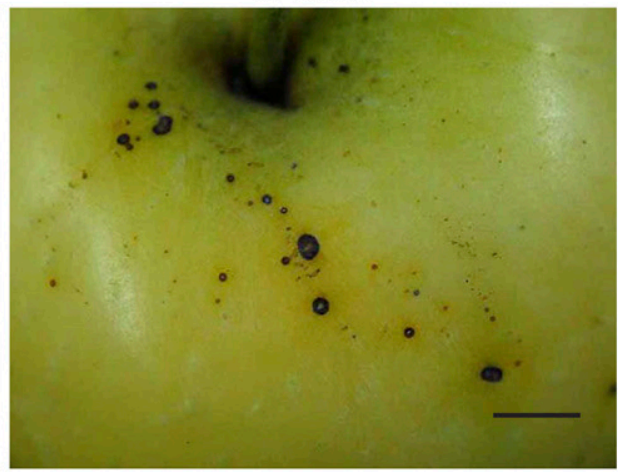

$150 \mathrm{dafb}$

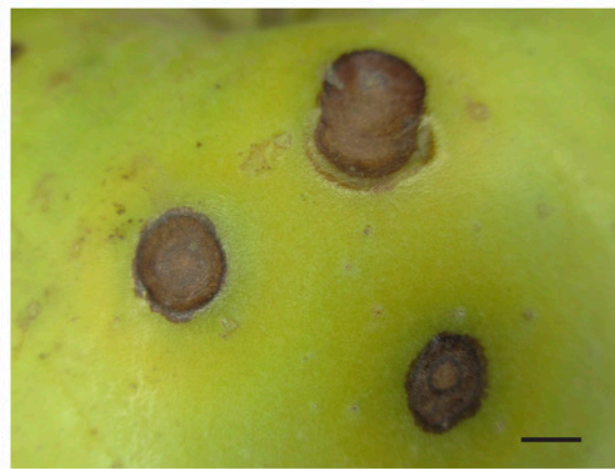

Fig. 5. Symptom development on apples that were inoculated with Trichothecium roseum at different ages after full bloom (dafb). A, Disease incidence (calculated as fruit infection rate) surveyed at 15 days postinoculation. Data points represent the mean incidence of black spots pooled from 2015 and 2016 field data. Bars indicate the standard error of the mean. Mean incidence for each year was based on examination of at least 30 independent fruit. Different lowercase letters on columns indicate significant difference $(P<0.05)$ based on Tukey's honest significant difference (HSD) mean separation test. B, Lesion diameters at harvest (165 day after full bloom). Each boxplot represents measurements from 40 independent Trichothecium black spot (TBS) lesions pooled over the 2015 and 2016 field experiments. Different lowercase letters on boxplots indicate significant difference $(P<0.05)$ based on Tukey's honest significant difference (HSD) mean separation test. C, Typical symptoms at harvest of TBS inoculated at 60 dafb, 75 dafb, 105 dafb, and 150 dafb. Bar = 5 mm. 
be to select waterproof bags with better air exchange capabilities in order to shorten periods of high humidity inside the bags. A second step would be to reduce humidity, and improve ventilation and light conditions in orchards. Batzer et al. (2013) showed that pruning was an effective measure to reduce the risk of blemishing by sooty blotch and flyspeck fungi by decreasing orchard RH. In most orchards in
China, apple trees are overcrowded. We therefore suggest that increased pruning could improve air movement, hasten drying, and reduce periods of high RH. In our work, apple fruit were susceptible to T. roseum from fruit expansion to ripening stages, and fungicide access to fruit would not be possible due to presence of the fruit bags. We speculate that protectant fungicides or biological agents with
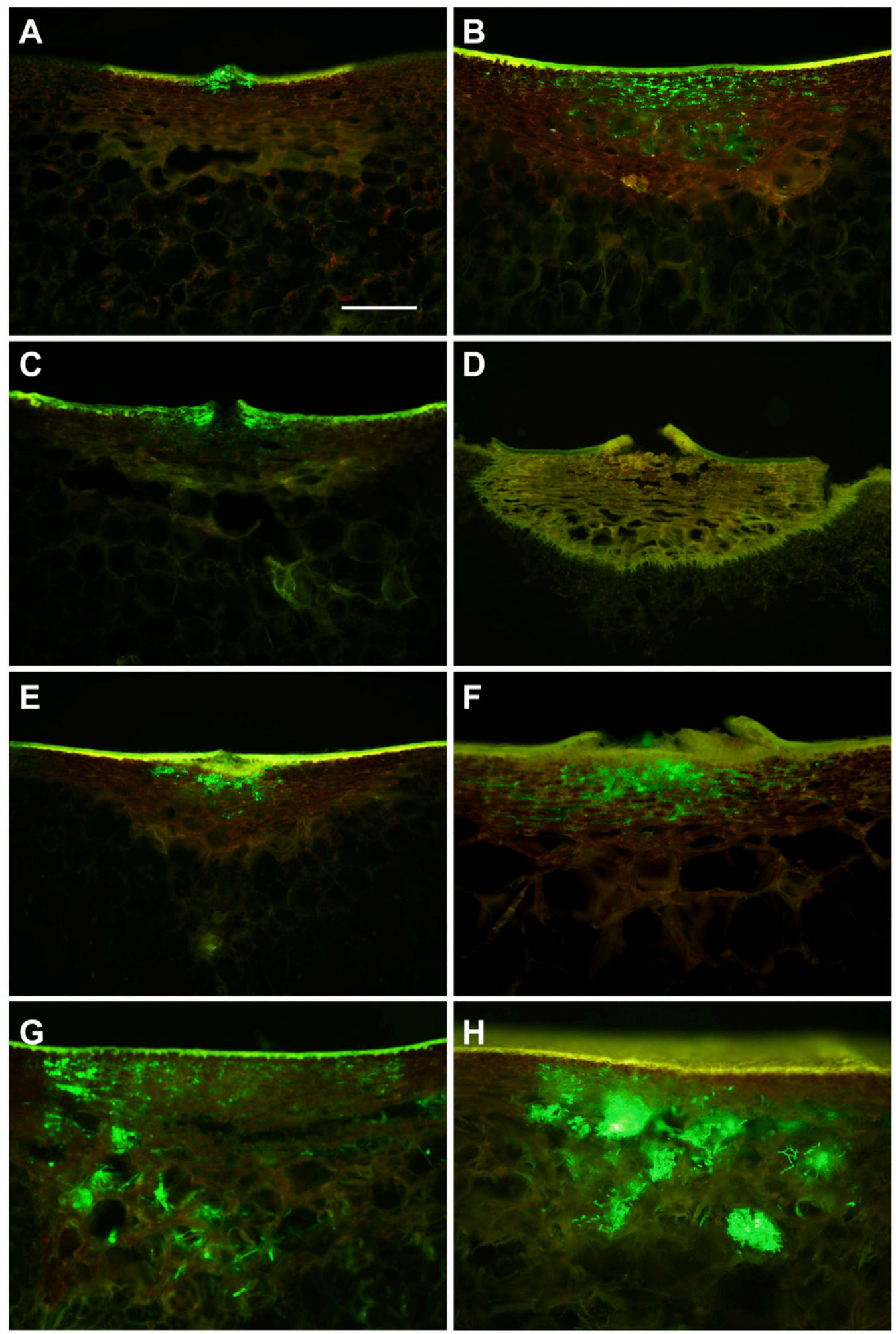

Fig. 6. Histological infection features of Trichothecium roseum conidia inoculated on apples at 75 days after full bloom (dafb), 105 dafb, and 150 dafb. A, B, C, D, 75 dafb. A, At 15 days postinoculation (dpi), hyphae colonized intensively under the stoma. B, At $30 \mathrm{dpi}$, most hyphae colonized hypodermal cell layers. C, At $60 \mathrm{dpi}$, green hyphae remained at openings, and hyphae in hypodermal cell layers were reduced. D, At 70 dpi, a complete lignification zone was formed around black spot tissues. E, F, G, 105 dafb. E, At 15 dpi, hyphae colonized dead tissues under lenticels. F, At 30 dpi, hyphae colonized hypodermal cell layers. G, At 60 dpi, hyphae extended to parenchymal cell layers. H, 150 dafb. At 15 dpi (around the harvest time), hyphae had intensively colonized parenchymal cells. Bar $(A-G)=200 \mu \mathrm{m}$. 

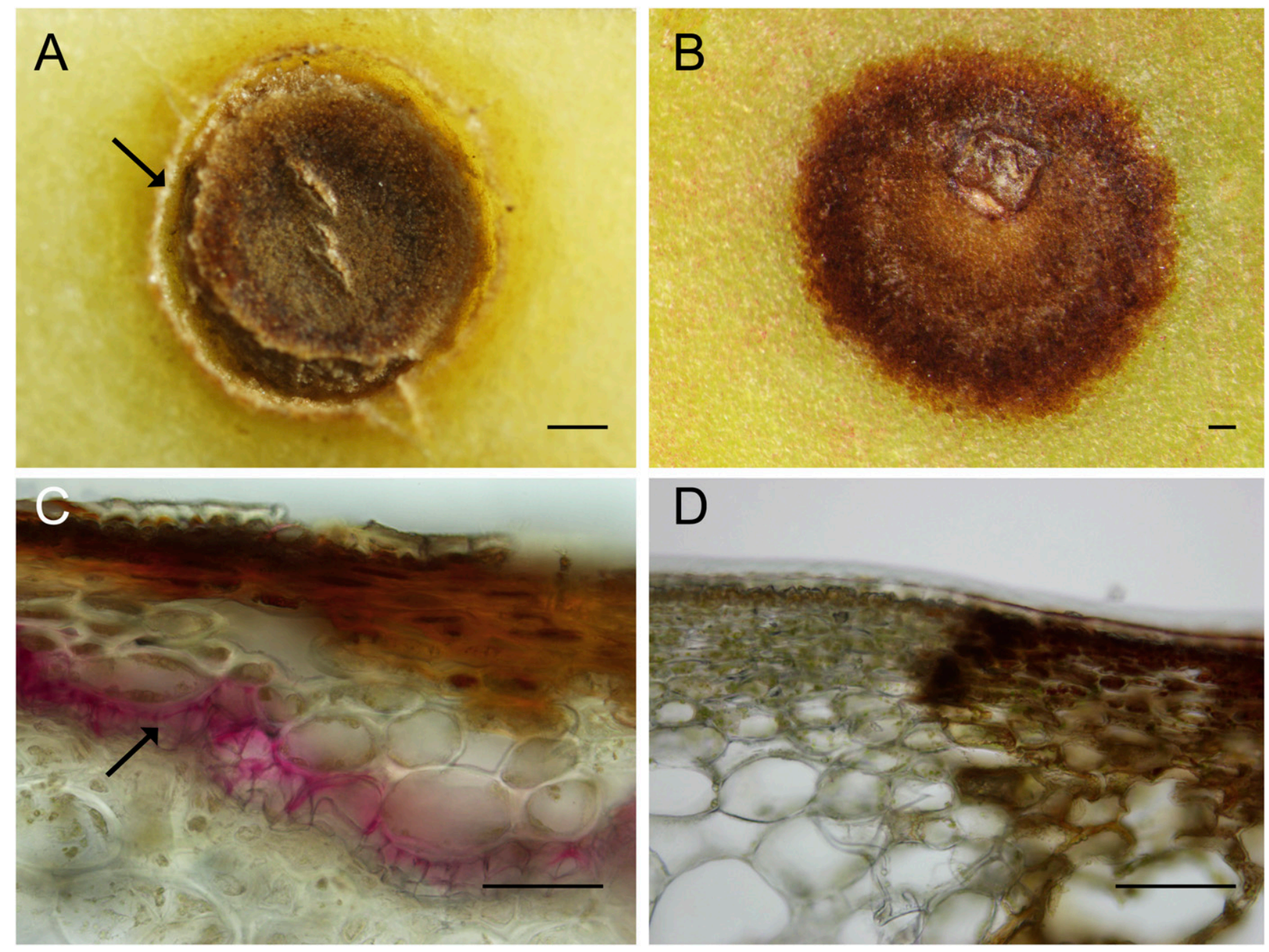

Fig. 7. Trichothecium black spot-induced host defense reactions observed on fruit inoculated at 60 (A, C) and 150 days after full bloom (dafb) (B, D). A, B, Separation layer that had formed in host tissue at harvest (165 dafb) (A, black arrow) was not evident in B. C, D, Differences in lignin deposition (based on phloroglucinol-hydrochloric acid staining) observed in $C$ (purple staining indicated by black arrow) but not in $D$ at harvest. $B a r(A-D)=100 \mu \mathrm{m}$.

long residual efficacy could be applied within 1 day before fruit bagging in order to reduce $T$. roseum.

Fruit bagging can efficiently prevent infection by many pathogens, such as Colletotrichum spp. causing bitter rot and Botryosphaeria spp. causing ring rot. But $T$. roseum can exist on sepals and floral residues on young apple fruit (Chen et al. 2002). We speculated that $T$. roseum thalli, existing on sepals and floral residues before fruit bagging, could be inocula for TBS. This also could partly explain why most black spots occur around sepals.

$T$. roseum can cause many diseases on diverse hosts with different symptoms, such as apple core rot (Gao et al. 2013), pink mold rot on apple, orange, tomato (Hamid et al. 2014), avocado (Sharma et al. 2016), peach (Hong and Michailides 1997), and strawberry (Kwon et al. 2010), and leaf spot on Mangifera indica L. (He et al. 2011). Whether there are pathogenicity differences is still unknown.

\section{Acknowledgments}

We thank Prof. Tom Hsiang, Department of Environmental Biology, University of Guelph, for comments, revisions and suggestions during manuscript preparation.

\section{Literature Cited}

Batzer, J. C., McManus, P. S., and Gleason, M. L. 2013. Fungicide spray volume and pruning impact performance of a warning system for sooty blotch and flyspeck on apple. Plant Health Prog. 14.

Belding, R. D., Sutton, T. B., Blankenship, S. M., and Young, E. 2000. Relationship between apple fruit epicuticular wax and growth of Peltaster fructicola and Leptodontidium elatius, two fungi that cause sooty blotch disease. Plant Dis. 84:767-772.

Chen, C., Wang, J. Y., Bao, Y. Y., and Bian, X. R. 2002. Investigation of occurrence and control of fruit spot of bagging apple. China Fruits (3):40-42.

Feng, F. J., Li, M. J., Ma, F. W., and Cheng, L. L. 2014. The effects of bagging and debagging on external fruit quality, metabolites, and the expression of anthocyanin biosynthetic genes in 'Jonagold' apple (Malus domestica Borkh.). Sci. Hortic. (Amsterdam) 165:123-131

Gao, L. L., Zhang, Q., Sun, X. Y., Jiang, L., Zhang, R., Sun, G. Y., Zha, Y. L., and Biggs, A. R. 2013. Etiology of moldy core, core browning, and core rot of Fuji apple in China. Plant Dis. 97:510-516.

Guo, J. M. 2013. Occurrence of black spot on bagged apple in Gansu Tianshui and integrated control strategy. China Fruits (2):56-58.

Guo, Y. Z., Sun, G. Y., Gao, B. W., Li, C. Y., Zhang, P. L., and Lei, X. L. 2005 Studies on the identification of pathogen and the biological characteristics of black-dot disease of bagged apple. Acta Agric. Boreali-Occidentalis Sin. 14(3):18-21.

Hamid, M. I., Hussain, M., Ghazanfar, M. U., Raza, M., and Liu, X. Z. 2014. Trichothecium roseum causes fruit rot of tomato, orange, and apple in Pakistan. Plant Dis. 98:1271.

Harris, R. F., Gardner, W. R., Adebayo, A. A., and Sommers, L. E. 1970. Agar dish isopiestic equilibration method for controlling the water potential of solid substrates. Appl. Microbiol. 19:536-537.

He, S. L., Liu, Z. L., Liu, X. M., Xie, Y. X., and Pu, J. J. 2011. A new disease in mango caused by Trichothecium roseum. J. Fruit Sci. 28:474-478.

Hong, C. X., and Michailides, T. J. 1997. Prune, plum, and nectarine as hosts of Trichothecium roseum in California orchards. Plant Dis. 81:112.

Hwang, B. K. 1983. Contents of sugars, fruit acids, amino acids and phenolic compounds of apple fruits in relation to their susceptibility to Botryosphaeria ribis. J. Phytopathol. 108:1-11.

Kohn, F. C., and Hendrix, F. F. 1983. Influence of sugar content and pH on development of white rot on apples. Plant Dis. 67:410-412. 
Kosuge, T., and Hewitt, W. B. 1964. Exudates of grape berries and their effect on germination of conidia of Botrytis cinerea. Phytopathology 54:167-172.

Kwon, J. H., Kim, M. J., Shim, C. K., Jee, H. J., and Lee, S. D. 2014. Pink mold rot on apple (Malus pumila var. dulcissima Koidz.) caused by Trichothecium roseum (Pers.) Link ex Gray in Korea. Korean J. Pestic. Sci. 18:429-433.

Kwon, J. H., Shen, S. S., and Kim, J. 2010. Occurrence of pink mold rot of strawberry caused by Trichothecium roseum in Korea. Plant Pathol. J. 26:296.

Li, E. M., Shi, D. C., Xu, Y. H., Chen, F., and Zhai, H. 2008. Changing characteristics of temperature and humidity in different type bags for bagging apple and their effects on fruit appearance quality. Chin. J. Appl. Ecol. 19:208-212.

Li, X. M., Li, J., Li, Q. L., Huang, J. B., Meng, L. F., and Yu, J. 2015. Effects of rainfall in different months on bagged apple black-rot disease. Chin. Agric. Sci. Bull. 31(4):217-221.

Liu, Q., and Xiao, C. L. 2005. Influence of nutrient and environmental factors on conidial germination of Potebniamyces pyri. Phytopathology 95:572-580.

Liu, Y. L., Fan, C. H., Che, F., Hui, M., Zhao, Z. Y., and Jiang, H. X. 2007. Occurrences of black-dot disease on bagged apple in Shaanxi province. J. Northwest For. Univ. 22(3):116-119.

Sharma, G., Maymon, M., and Freeman, S. 2016. First detailed report of Trichothecium roseum causing post-harvest pink rot of avocado in Israel. Plant Dis. 100:856.

Sitterly, W. R., and Shay, J. R. 1960. Physiological factors affecting the onset of susceptibility of apple fruit to rotting by fungus pathogens. Phytopathology 50:91-93.
Wang, B., Li, B. H., Dong, X. L., Wang, C. X., and Zhang, Z. F. 2015. Effects of temperature, wetness duration, and moisture on the conidial germination, infection, and disease incubation period of Glomerella cingulata. Plant Dis. 99:249-256.

Wang, C. C., Jin, J., Li, B. H., Wang, C. X., Dong, X. L., and Zhang, Z. F. 2014 Pathogens isolated from different symptoms of black spot disease and infection conditions. Acta Agric. Boreali Sin. 29:136-144

Wang, J. Y., Wang, S. M., and Gao, H. J. 2006. Review on research progress on the control of apple diseases and insect pests by bagging. Chin. Agric. Bull. 22(8):423-426

Wen, Y. Q., and Ma, F. W. 2006. An advance in application and study of apple bagging technology in China. J Northwest A\&F University (Nat. Sci. Ed.) 34 (2):100-104

Wrona, B., and Grabowski, M. 2004. Influence of fructose and glucose occurring on fruit surface on the growth of fungi that cause sooty blotch of apple. J. Plant Prot. Res. 44:287-292.

Xu, X. M., Guerin, L., and Robinson, J. D. 2001. Effects of temperature and relative humidity on conidial germination and viability, colonization and sporulation of Monilinia fructigena. Plant Pathol. 50:561-568.

Yukita, K., and Kuradate, K. 1988. Pink rot caused by Trichothecium roseum on bagged apple fruits. Ann. Phytopathol. Soc. Jpn. 64:610.

Zhang, Y. Z., Li, P. M., and Cheng, L. L. 2010. Developmental changes of carbohydrates, organic acids, amino acids, and phenolic compounds in 'Honeycrisp' apple flesh. Food Chem. 123:1013-1018. 